\title{
Nachrufe
}

Chirurg 2019 - 90:419-420

https://doi.org/10.1007/s00104-019-0964-7

(c) Springer Medizin Verlag GmbH, ein Teil von Springer Nature 2019
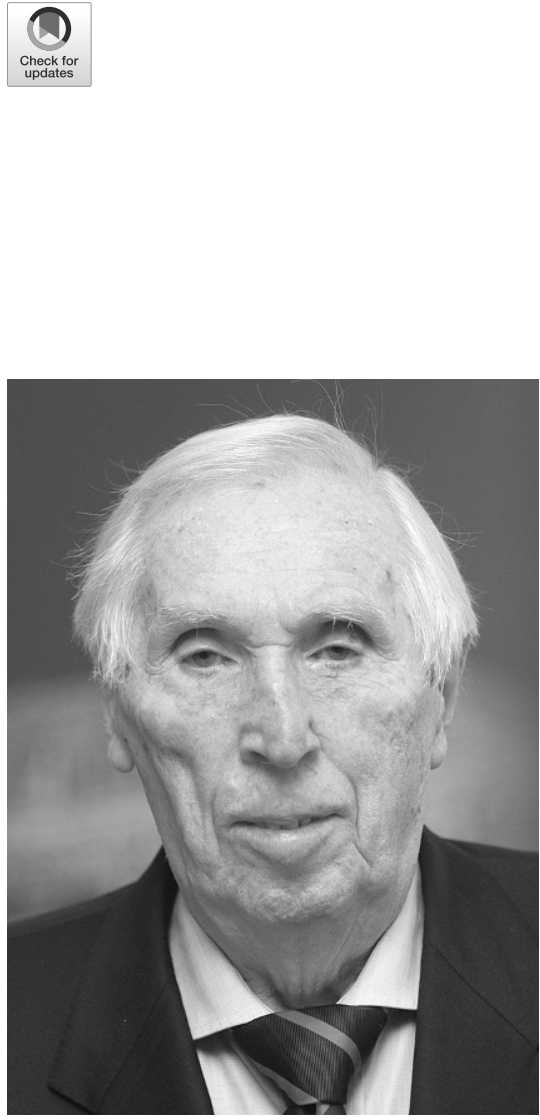

In memoriam Prof. h. c. Dr. med. Karl Hempel (12.06.1923 bis 07.12.2018)

Am 07.12.2018 verstarb im 96. Lebensjahr der langjährige Präsident und Ehrenpräsident des Berufsverbandes der Deutschen Chirurgen (BDC), Professor h.c. Dr. med. Karl Hempel. Wie kein Zweiter hat Prof. Hempel die Geschicke des BDC in einer äußerst bewegten Zeit mit Ruhe und Besonnenheit bestimmt. Wir gedenken seiner mit tief empfundener Hochachtung.

Karl Hempel („der Preuße“) wurde am 12.06.1923 in Kolberg/Pommern geboren, absolvierte das Abitur am humanistischen Domgymnasium, anschließend das Medizinstudium in Berlin und Würzburg mit Unterbrechungen durch Wehrdienst und Fronteinsatz. 1946 folgte der

Dieser Nachruf wurde erstpubliziert in Passion Chirurgie (2019) 9(3):41-42.

\author{
J.-A. Rüggeberg ${ }^{1,2} \cdot$ J. Seifert ${ }^{1} \cdot$ H.-J. Meyer ${ }^{1,3}$ \\ 'Berufsverband der Deutschen Chirurgen e. V. (BDC), Berlin, Deutschland \\ ${ }^{2}$ Chirurgische Praxis Dr. Rüggeberg, Ottersberg, Deutschland \\ ${ }^{3}$ Deutsche Gesellschaft für Chirurgie e. V. (DGCH), Berlin, Deutschland
}

\section{Nachruf zum Tod von Prof. h.c. Dr. med. Karl Hempel}

Abschluss mit Staatsexamen und Promotion in Hamburg. Nach Assistenz- und Oberarztjahren wurde er 1969 als Chefarzt der Chirurgischen Abteilung am AK Hamburg Wandsbek gewählt und war dort zusätzlich als Ärztlicher Direktor bis zu seiner Pensionierung 1988 tätig.

Während seiner chirurgischen Tätigkeit unternahm er zahlreiche Studienreisen ins Ausland, u.a. nach Kopenhagen, Chur, London, Glasgow und vor allem in die USA (Harvard, New York, Newark und North Carolina). 1982, also noch während seiner aktiven Zeit, wurde Karl Hempel vom Präsidium des BDC einstimmig als Nachfolger von Prof. MüllerOsten zum Präsidenten gewählt, ein Amt, das er über vier Wahlperioden bis 1998 ausübte. Bei der Amtsübergabe an seinen Nachfolger Prof. Witte wurde Karl Hempel wiederum einstimmig vom geschäftsführenden Präsidium des BDC zum Ehrenpräsidenten ernannt. Im November 1998 wird ihm neben der Ehrenmitgliedschaft die Ehrenmedaille des BDC verliehen. $\mathrm{Zu}$ seinem 95. Geburtstag durften wir ihm die Wolfgang-Müller-OstenMedaille noch persönlich in Hamburg überreichen.

Seine Präsidentschaft wird immer verbunden sein mit dem Ausbau des BDC zur größten Chirurgenvereinigung Europas und der klaren Aufgabentrennung eines Berufsverbandes gegenüber wissenschaftlichen Fachgesellschaften. Von ihm stammt das prägende Wort: „Die Gesellschaft ist für die Chirurgie da, der BDC für die Chirurgen!“ In seiner Amtszeit nahm die Mitgliederzahl des BDC von 3400 (1982) auf 12.539 (1998) zu.

Besonders bewegend für den „Preußen“ Karl Hempel war die Wiederver- einigung Deutschlands 1989. Sobald wie möglich wurden die Chirurgen in der ehemaligen DDR besucht und mit „Rat und Tat", vor allem durch Fachbücher und Zeitschriften, unterstützt. Viele Kollegen konnten für die Mitgliedschaft und Mitarbeit im BDC gewonnen werden und wurden $\mathrm{zu}$ persönlichen Freunden. Vorbildhaft ist Karl Hempel dabei vorurteilsfrei mit selbstverständlicher und menschlicher Art mit den oft schwierigen Biographien der Kollegen umgegangen.

Ein weiterer Meilenstein seiner Präsidentschaft war die Gründung der „Akademie für Chirurgische Weiterund praktische Fortbildung", heute die größte Akademie für die Durchführung zahlreicher Seminare zur Fortund Weiterbildung für junge Chirurgen, für Postgraduierte sowie leitende Krankenhausärzte und Niedergelassene.

Der Ausbau der Rechtsberatung und vor allem eine enge Zusammenarbeit mit dem Vorstand und Präsidium der Deutschen Gesellschaft für Chirurgie (DGCH), deren Ehrenmitglied er war, haben den Mitgliedern des BDC den Wert des Verbandes verdeutlicht sowie mögliche Animositäten und Reibungsverluste erfreulicherweise deutlich minimiert.

Karl Hempel leitete die Sitzungen des BDC souverän, ruhig, jedoch scharfen Bemerkungen nicht abgeneigt („völliger Blödsinn!“). Langatmige Stellungnahmen waren ihm ein Gräuel; der aufmerksame Beobachter merkte es an den „restless legs“. Objektive Kritik, die ihm subjektiv überzogen schien, ließ er zu und in die Entscheidungen einfließen, allerdings „contre cœur“. 


\section{Nachrufe}

Karl Hempel war stets bescheiden und zurückhaltend, aber bestimmt in seinem Auftreten. Alles Extrovertierte oder gar Bombastische war ihm fremd, dabei vermochte er durchaus mit einem gewissen Schalk sein Gegenüber auf das rechte $\mathrm{Maß}$ zu bringen. Eine kleine Anekdote mag dies verdeutlichen: Ein aufgebrachter Kollege kam in die Hamburger Geschäftsstelle, um sofort eine Lösung für sein Problem zu bekommen. Hempel bat ihn auf das etwas abgenutzte Sofa und ließ ihn reden, bis alles gesagt war, ohne selber einzugreifen. Am Ende stand der Kollege auf, bedankte sich überschwänglich und verließ das Büro in der Überzeugung, tatkräftige Hilfe gefunden zu haben, ohne dass sich an der Ausgangslage irgendetwas geändert hätte.

Alle, die ihn erleben durften, ob Schüler, Mitarbeiter, Mitstreiter, Kollegen oder Freunde, respektierten und schätzten ihn und seine unkomplizierte, gradlinige, humorvolle Art. Wir werden seinen Ausspruch: „Chirurgie muss dem Patienten nutzen und nicht dem Chirurgen" immer als Leitspruch bewahren und sein Andenken in dauerhaften Ehren halten.

Karl Hempel hat sich um die deutsche Chirurgie und die deutschen Chirurgen verdient gemacht, die Historie des BDC ist ohne ihn nicht vorstellbar.

Prof. Dr. med. Dr. h.c. Hans-Joachim Meyer

Präsident des Berufsverbandes der Deutschen Chirurgen e.V. (BDC)

Generalsekretär der Deutschen Gesellschaft für Chirurgie e.V. (DGCH)

Dr. med. Jörg-Andreas Rüggeberg Vizepräsident des Berufsverbandes der Deutschen Chirurgen e. V. (BDC)

Prof. Dr. med. Julia Seifert Vizepräsidentin des Berufsverbandes der Deutschen Chirurgen e. V. (BDC)

\section{Korrespondenzadresse}

Dr. med. J.-A. Rüggeberg

Chirurgische Praxis Dr. Rüggeberg

Am Damm 8, 28870 Ottersberg, Deutschland

dr.rueggeberg@t-online.de 